\title{
SOME INFINITE FREE BOUNDARY PROBLEMS
}

\author{
BY
}

DAVID E. TEPPER AND GERALD WILDENBERG

\begin{abstract}
Let $\Gamma$ be the boundary of an unbounded simply connected region $\mathscr{D}$, and let $e(\Gamma)$ denote the family of all simply connected regions $\Delta \subset \mathscr{D}$ such that $\partial \Delta=\Gamma \cup \gamma$ where $\gamma \cap \Gamma$ contains only the infinite point. For $\Delta \in e(\Gamma)$ we call $\gamma$ the free boundary of $\Delta$. Given a positive constant $\lambda$, we seek to find a region $\Delta_{\lambda} \in \mathcal{C}(\Gamma)$ with free boundary $\gamma_{\lambda}$ such that there is a bounded harmonic function $V$ in $\Delta_{\lambda}$ with the properties that (i) $V=0$ on $\Gamma$, (ii) $V=1$ on $\gamma$, (iii) $|\operatorname{grad} V(z)|=\lambda$ for $z \in \gamma_{\lambda}$. We give sufficient conditions for existence and uniqueness of $\Delta_{\lambda}$. We also give quantitative properties of $\gamma_{\lambda}$.
\end{abstract}

1. Introduction. Suppose a connected set $\Gamma$ containing more than one point is the boundary of a region $\mathscr{D}$ which is simply connected on the Riemann sphere and is unbounded in the complex plane. We define $C(\Gamma)$ to be the class of regions $\omega$ with the following properties.

(a) $\partial \omega=\Gamma \cup \alpha$ where $\alpha$ is a nonempty connected subset of $\mathscr{D}$ which does not meet $\Gamma$ in the finite plane.

(b) If $\Gamma$ is compact, then $\alpha$ separates $\Gamma$ from the point at infinity.

(c) If $\Gamma$ is not compact, then $\alpha \cup \Gamma$ is connected on the Riemann sphere.

Given $Q$, a continuous positive function in $\mathscr{D}$, we seek a region $\omega \in \mathcal{C}(\Gamma)$ with the property that there is a bounded harmonic function $V_{\omega}$ in $\omega$ satisfying the following three conditions.

(1) $V_{\omega}=0$ on $\Gamma$,

(2) $V_{\omega}=1$ on $\alpha$,

(3) $\left|\operatorname{grad} V_{\omega}\right|=Q$ on $\alpha$.

For $\omega \in \mathcal{C}(\Gamma)$ the bounded harmonic function denoted $V_{\omega}$ satisfying conditions (1) and (2) will be called the stream function of $\omega$ and the set $\alpha$ will be called the free boundary of $\omega$. For the case where $\Gamma$ is compact, sufficient conditions for existence and uniqueness of a free boundary such that $V_{\omega}$ satisfies condition (3) are given in [1], [4], [5] and [6]. Also, qualitative properties of the free boundary $\alpha$ are given in these papers. It should be pointed out that many of the ideas are outgrowths of Beurling's existence theorem in [3].

Received by the editors June 14, 1977.

AMS (MOS) subject classifications (1970). Primary 31A05; Secondary 76G20.

Key words and phrases. Harmonic function, Dirichlet problem, free boundary.

(C) 1979 American Mathematical Society 0002-9947/79/0000-0056/\$03.50 
We say $\Gamma$ is starlike if for $w \in \Gamma, \rho w \notin \mathscr{D}$ for $0<\rho<1$. We will assume that $\Gamma$ is either starlike or in some sense is the limit of starlike curves. Although we will be mainly interested in the case where $Q$ is a positive constant, we will have to apply conformal mapping to reformulate the problem in order to obtain some of our results; hence the problem is stated in the more general form. We remark that many of our results remain true if we consider the case where $\Gamma$ is starlike and $\rho Q(\rho z)$ is nondecreasing for $0<\rho<\infty ;$ see [1].

Since results from [4], [5], and [6] will be frequently used, we now summarize some of the results which are either included in or are easy consequences of the results in those papers. Let $\Gamma$ be compact and $Q$ a positive constant which we denote by $\lambda$, then:

(i) There exists $\omega \in \mathcal{C}(\Gamma)$ such that $V_{\omega}$ satisfies (3) on $\alpha_{\lambda}$ the free boundary of $\omega$. Furthermore, $\alpha_{\lambda}$ is an analytic Jordan curve.

We say $\Gamma$ is convex if $\mathbf{C}-\mathscr{D}$ is a convex set.

(ii) If $\Gamma$ is convex, then $\alpha_{\lambda}$ is unique and convex, and for $z \in \alpha_{\lambda}$ the distance to $\Gamma$ denoted $d(z, \Gamma)$ is less than $1 / \lambda$.

(iii) If $\Gamma$ is starlike, then $\alpha_{\lambda}$ is unique and starlike.

(iv) If $\Gamma_{1}$ and $\Gamma_{2}$ are each starlike and are each the boundary of respective unbounded regions $\mathscr{D}_{1}$ and $\mathscr{D}_{2}$, with $\mathscr{D}_{1} \subset \mathscr{D}_{2}$ and if $\alpha_{1}$ and $\alpha_{2}$ are the respective free boundaries in (iii) corresponding to $\Gamma_{1}$ and $\Gamma_{2}$, then $\alpha_{2}$ lies inside $\alpha_{1}$.

In the cases where $\Gamma$ is convex or starlike then we call $\alpha_{\lambda}$ the solution free boundary for $\Gamma$. We will need the following result for the general problem. The proof is given in [4].

(v) Suppose $Q$ is continuous in $\mathscr{D}$ and as $z \in \mathscr{D}$ tends to $\Gamma$, let $Q$ have the property that:

$$
Q(z)=o\left(\frac{|\operatorname{grad} u|}{u}\right)
$$

where $u$ is any positive harmonic function defined in some $\Omega \in \mathcal{C}(\Gamma)$ and where $u=0$ on $\Gamma$. Then, if there is an $\omega^{\prime} \in \mathcal{C}(\Gamma)$ with the property that for all $z \in{ }^{\prime}$, for which

$$
\limsup _{\substack{\zeta \rightarrow z \\ \zeta \in \omega^{\prime}}} \frac{\left|\operatorname{grad} V_{\omega^{\prime}}(\zeta)\right|}{Q(\zeta)}<1,
$$

then there exists $\omega \in \mathcal{C}(\Gamma)$ such that $\omega \subset \omega^{\prime}$ and $V_{\omega}$ satisfies (1), (2) and (3).

2. Convexity. If $\Gamma$ is convex, then we use facts (i)-(v) to construct a solution which is unique in a certain sense. We begin with the following theorem.

THEOREM 1. If $\Gamma$ is convex and unbounded and $\lambda$ is a positive constant, then there exists $\omega \in \mathcal{C}(\Gamma)$ with the following properties: 
(a) $V_{\omega}$ satisfies (3) for $Q \equiv \lambda$.

(b) If $\alpha_{\lambda}$ is the free boundary of $\omega$, then $\alpha_{\lambda}$ is of distance less than $1 / \lambda$ from $\Gamma$.

Proof. Without loss of generality we suppose that $\Gamma$ is contained in the lower half plane and the origin lies on $\Gamma$. For $t>0$ let $H_{t}$ denote the half plane $\operatorname{Im} z \leqslant-t$ and $\mathscr{D}_{t}=H_{t} \cup \mathscr{D}$. The unbounded region $\mathscr{D}_{t}$ will have as its boundary the compact and convex set $\Gamma_{t}$. Let $\omega_{t}$ be the element of $e\left(\Gamma_{t}\right)$ such that $\alpha_{\lambda}^{(t)}$, the free boundary of $\omega_{t}$, is the solution free boundary for $\Gamma_{t}$. Existence and uniqueness of $\omega_{t}$ is asserted in facts (i) and (ii). We claim that

$$
\omega=\bigcup_{t>0}\left(\omega_{t} \cap \mathscr{D}\right)
$$

satisfies (a) and (b). We note that (b) follows easily from (iv) and (ii). It remains to show (a).

We see by (iv) that for $t>s$ we have $\alpha_{\lambda}^{(s)}$ lies inside $\alpha_{\lambda}^{(t)}$. Furthermore, for $s>t$, (a) if $z \in \omega_{s} \cap \mathscr{D}$, then $z \in \omega_{t} \cap \mathscr{D}$ and (b) $V_{\omega_{t}}(z)$ is nonincreasing with $t$. Therefore, as $t \rightarrow \infty, V_{\omega_{t}}(z) \rightarrow V_{\omega}(z)$. Furthermore, if $z \in \alpha$, the free boundary of $\omega$, then every neighborhood of $z$ contains points from all but finitely many of the $\left\{\alpha_{t}\right\}$. Thus $\left|\operatorname{grad} V_{\omega}(z)\right|=\lambda$.

For $\Gamma$ unbounded and convex, we will call the region $\omega \in \mathcal{C}(\Gamma)$ from Theorem 1 the construction solution strip. We will call its free boundary the constructed free boundary. Any other $\omega \in C(\Gamma)$ where $V_{\omega}$ satisfies (1), (2) and (3) will be called a solution. In order to prove that the constructed solution is minimal, we will need the following lemma.

LEMMA 1. Let $\Gamma$ be convex and compact. Suppose there exists $\omega^{*} \in \mathcal{C}(\Gamma)$ whose free boundary $\alpha^{*}$ is the union of finitely many analytic arcs. Suppose for each $z$ interior to one of these arcs we have

$$
\lim _{\substack{\zeta \rightarrow z \\ \zeta \in \omega^{*}}}\left|\operatorname{grad} V_{\omega^{\prime}}(z)\right| \leqslant \lambda .
$$

Then, $\omega^{*}$ contains the solution free boundary for $\Gamma$.

Proof. Let $f$ be a mapping of $|w|>1$ onto $\mathscr{D}$ and let $\tilde{\Gamma}=f^{-1}(\Gamma)$. We consider the general problem for the set $\tilde{\Gamma}$ which is the boundary of the region $\tilde{\mathscr{D}},|w|>1$, and the positive function $Q(w)=\lambda\left|f^{\prime}(w)\right|$. The region $\tilde{\omega}^{*}=$ $f^{-1}\left(\omega^{*}\right)$ satisfies condition (4) at all but finitely many points on $\tilde{\alpha}^{*}=$ $f^{-1}\left(\alpha^{*}\right)$. For $|w|<1$, define $Q(w)=Q(1 / \bar{w})$ and let $\tilde{\Omega}^{*}$ be the region obtained by reflecting $\tilde{\omega}^{*}$ about the circle $|w|=1$. Because of the regularity condition on the boundary of $\tilde{\Omega}^{*}$, if $H(w)$ solves the Dirichlet problem in the region $\tilde{\Omega}^{*}$ with boundary values $\log Q(w)$, and if $g(\zeta, w)$ is the Green's 
function of $\tilde{\Omega}^{*}$ we have:

$$
\begin{aligned}
\log \left|\operatorname{grad} V_{\tilde{\omega}^{*}}(w)\right| & =\frac{1}{2 \pi} \int_{\partial \tilde{\omega}^{*}} \log \left|\operatorname{grad} V_{\tilde{\omega}^{*}}(\zeta)\right| \frac{\partial g(w, \zeta)}{\partial \eta} d s \\
& \leqslant \frac{1}{2 \pi} \int_{\partial \tilde{\omega}^{*}} H(\zeta) \frac{\partial g(w, \zeta)}{\partial \eta} d s=H(w)
\end{aligned}
$$

where $\partial / \partial \eta$ represents differentiation with respect to the inward pointing normal. From this we obtain (4) so $\omega^{*}$ contains $\alpha_{\lambda}$ the unique free boundary solution for $\Gamma$.

We now prove that the constructed solution is minimal.

THEOREM 2. Let $\Gamma$ be an unbounded convex curve. Suppose $Q(z) \equiv \lambda$, a positive constant and $\omega^{\prime} \in \mathcal{C}(\Gamma)$ has its stream function $V_{\omega^{\prime}}$ which satisfies (1), (2) and (3). Then, $\omega^{\prime}$ contains $\omega$, the constructed solution strip.

PRoof. It follows from the Schwarz reflection principle that the free boundary of $\omega^{\prime}$ which we denote by $\alpha^{\prime}$ is an analytic curve. Using the notation of Theorem 1, we show $\omega_{t} \cap \mathcal{D}$ is contained inside $\omega^{\prime}$ for all $t$ by supposing there is some $t$ such that $\omega_{t} \cap \mathscr{D}$ does not lie inside $\omega^{\prime}$, and getting a contradiction. In the family $\mathcal{C}\left(\Gamma_{t}\right)$ consider the annulus $\omega^{*}$ which consists of all points $z$ which can be connected to $\Gamma_{t}$ by an arc which lies in the set

$$
\left[\omega_{t} \cap \omega^{\prime}\right] \cup\left[\omega_{t} \cap(C-\mathscr{D}) \cap H_{t}\right] \text {. }
$$

Since $\alpha_{t}$ and $\alpha^{\prime}$ are each analytic curves, it follows that the free boundary of $\omega^{*}$ consists of finitely many analytic arcs. Furthermore since $V_{\omega^{*}}(z) \geqslant V_{\omega^{\prime}}(z)$ and $V_{\omega^{*}}(z) \geqslant V_{\omega_{t}}(z)$ for $z \in \omega^{*}$,

$$
\left|\operatorname{grad} V_{\omega^{*}}(z)\right| \leqslant \lambda
$$

at all regular points on the free boundary of $\omega^{*}$. This implies that $\omega^{*}$ contains $\omega_{t}$ which in turn implies $\omega^{\prime}$ contains $\omega_{t} \cap \mathcal{D}$ for all $t>0$ which is our desired contradiction.

We now wish to study the asymptotic behavior of the constructed solution in the case where $\Gamma$ is convex. To do this we assume $\Gamma$ is continuously parametrized by the equation $z=z(t),-\infty<t<\infty$, in the following manner;

$$
z(0)=0 \text {. }
$$

If $\langle z(t)\rangle$ is the signed arc length from 0 to $z(t)$, then $t\langle s$ implies that $\langle z(t)\rangle$ is less than $\langle z(s)\rangle$.

$$
\begin{gathered}
\lim _{t \rightarrow \infty}|z(t)|=\lim _{t \rightarrow \infty}|z(t)|=\infty . \\
\operatorname{Re} z(t)>0 \text { for } t>0 .
\end{gathered}
$$

We will also need the following notation. If $\beta$ is a curve and $z \in \mathbf{C}$, then 
$d(z, \beta)$ is the distance from $z$ to $\beta$. If the ray $r$ from the point $z \in \mathbf{C}$ intersects the curve $\beta$ at a furthest point $\zeta \in \beta$, then the distance from $z$ to $\beta$ along $r$ is defined as:

$$
d(z, \beta ; r)=|z-\zeta| \text {. }
$$

We are now ready to give qualitative properties of the constructed solution strip.

THEOREM 3. Let $\Gamma$ be unbounded and convex curve which is parametrized by $z=z(t)$ which satisfies (7), (8), (9) and (10) for $-\infty<t<\infty$. Let $l_{1}$ and $l_{2}$ be two straight lines such that

$$
\lim _{t \rightarrow \infty} d\left(z(t), l_{1}\right)=\lim _{t \rightarrow-\infty} d\left(z(t), l_{2}\right)=0 .
$$

If $\alpha$ is the free boundary of the constructed solution strip, then the free boundary of any other solution is not asymptotic to $\alpha$.

Proof. We suppose $\Gamma$ is contained in the lower half plane $H$ and $r_{1}$ and $r_{2}$ be the rays defined by $r_{i}=l_{i} \cap H$ for $i=1,2$. Since $\Gamma$ is convex and for $z \in \alpha, d(z, \Gamma)<1 / \lambda$, we must have $\alpha$ asymptotic to a pair of rays $\hat{r}_{1}$ and $\hat{r}_{2}$ with $\hat{r}_{i}$ parallel to $r_{i}$ for $i=1,2$. Let $\omega^{\prime}$ be a solution with free boundary $\alpha^{\prime}$ which is asymptotic to $\alpha$. Since $\alpha$ is minimal, we know that $\alpha$ separates $\alpha^{\prime}$ from $\Gamma$. For $i=1,2$, given $\varepsilon>0$ let $r_{i, \varepsilon}$ and $R_{i, e}$ be the pair of rays from the real axis which are parallel to $\hat{r}_{i}$ and are at a distance $\varepsilon$ from the ray $\hat{r}_{i}$ with $r_{i, \varepsilon}$ separating $\alpha$ from $\Gamma$. Let $c$ and $d$ be two rays in $H$, originating at 0 , which intersect $R_{i, e}$ and $r_{i, \varepsilon}$ for $i=1,2$. Since $R_{i, \varepsilon}$ and $r_{i, e}$ are parallel, we observe that

$$
\frac{d\left(0, r_{i, \mathrm{e}} ; c\right)}{d\left(0, R_{i, \mathrm{e}} ; c\right)}=\frac{d\left(0, r_{i, \mathrm{e}} ; d\right)}{d\left(0, R_{i, \mathrm{e}} ; d\right)}
$$

If the ray $c$ has a slope sufficiently close to the slope of $\hat{r}_{i}$, we have

$$
d\left(0, R_{i, \varepsilon} ; c\right) \geqslant d\left(0, \alpha^{\prime} ; c\right) \geqslant d(0, \alpha ; c) \geqslant d\left(0, r_{i, \varepsilon} ; c\right) \text {. }
$$

Now if $\varepsilon^{\prime}$ is a sufficiently small positive number and the slope of $c$ is sufficiently close to the slope of $\hat{r}_{i}$, then we have

$$
1-\varepsilon^{\prime}<\frac{d\left(0, r_{i, e} ; c\right)}{d\left(0, R_{i, e} ; c\right)}<1 .
$$

But then if the slope of $c$ is sufficiently close to the slope of $\hat{r}_{i}$, we have:

$$
1-\varepsilon^{\prime}<\frac{d(0, \alpha ; c)}{d\left(0, \alpha^{\prime} ; c\right)} \leqslant 1 .
$$

Let $\hat{R}$ be the collection of all rays through $z=0$. If

$$
M=\inf _{c \in \hat{R}} \frac{d(0, \alpha ; c)}{d\left(0, \alpha^{\prime} ; c\right)},
$$


then we see that $M<1$ and that $M \alpha^{\prime}=\left\{w: w=M z, z \in \alpha^{\prime}\right\}$ lies inside $\alpha$. We claim that $M \alpha^{\prime}$ is tangent to $\alpha$ at some point $z \in \alpha$. Suppose this is not the case. Relation (16) assures us that given $\delta>0$ there exists $\rho>0$ such that if $z^{\prime} \in \alpha^{\prime}$ and $\left|z^{\prime}\right|>\rho$, then if $z \in \alpha$ lies on the line connecting $z^{\prime}$ to the origin we have $\left|z / z^{\prime}\right|>M+\delta$. Now suppose for $z^{\prime} \in \alpha^{\prime}$ with $\left|z^{\prime}\right|<\rho$ we have $\left|M z^{\prime}-z\right|>0$ where $z \in \alpha$ lies on the line connecting $z^{\prime}$ to the origin. Then, we have $\left|z / z^{\prime}\right|<M$ for $\left|z^{\prime}\right|<\rho$. Thus we see there exists $z^{\prime} \in \alpha^{\prime}$ where $M z^{\prime} \in \alpha$. From this it follows that $V_{\omega^{\prime}}\left(M^{-1} z\right) \geqslant V_{\omega}(z)$ for $z \in \omega \cap M \omega^{\prime}$. Taking normal derivatives to $\alpha$ at $M z^{\prime}$, we get $M^{-1} \lambda<\lambda$ which is a contradiction.

The next theorem gives information about the asymptotic behavior of the free boundary.

THEOREM 4. Suppose $\Gamma$ is parametrized by $z=z(t)$ which satisfies (7), (8), (9), (10) and (12). Then

$$
\lim _{t \rightarrow \infty} d(z(t), \alpha)=\lim _{t \rightarrow-\infty} d(z(t), \alpha)=1 / \lambda .
$$

Proof. We define a monotone function for $-\infty<t<\infty$ by letting $\theta(t)=\theta(z(t))$ be the acute angle between any line of support for $\Gamma$ through $z(t)$ and the $x$-axis. Where there is more than one line of support, one may choose one arbitrarily. Define

$$
\theta_{1}=\sup _{-\infty<t<\infty} \theta(t), \quad \theta_{2}=\inf _{-\infty<t<\infty} \theta(t) .
$$

The first portion of this proof is the demonstration of the following statement:

FACT A. For any $N>0, \varepsilon>0$ there exists $t_{0}>0$ such that for all $t$ with $|t|>t_{0}$ there is a chord $L$ of length $N$ with the distance from $z(t)$ to the midpoint of $L$ less than $\varepsilon$.

In proving Fact $\mathrm{A}$, we first assume $s>0$; the corresponding statements about $s<0$ are essentially equivalent. Let $\hat{s}$ be chosen so that

$$
\left|\int_{s}^{\infty} d \theta(s)\right|<\delta
$$

where $\delta$ is as yet unspecified. The existence of such an $\hat{s}$ depending on $\delta$ is clear from the convexity of $\Gamma$. Let $s^{\prime}$ be chosen so that

$$
\left|z(\hat{s})-z\left(s^{\prime}\right)\right|>N
$$

and $s^{\prime}>\hat{s}$.

Consider a chord $L$ of length $N$ with endpoints $z_{1}=z\left(s_{1}\right)$ and $z_{2}=z\left(s_{2}\right)$ where $s_{2}>s_{1}>s^{\prime}$. Let $L_{1}$ and $L_{2}$ be lines of support of $\Gamma$ at $z_{1}$ and $z_{2}$ respectively and consider the (possibly degenerate) triangle formed by the lines $L, L_{1}$ and $L_{2}$. Let $L_{3}$ be the straight line carrying the segment $L$. If $M_{1}$, 
$M_{2}$ and $M_{3}$ are the respective slopes of $L_{1}, L_{2}$ and $L_{3}$, then

$$
\delta \geqslant\left|\theta_{1}-\arctan M_{1}\right| \geqslant\left|\theta_{1}-\arctan M_{3}\right| \geqslant\left|\theta_{1}-\arctan M_{2}\right| \geqslant 0
$$

because $M_{2} \leqslant M_{3} \leqslant M_{1}$. Therefore, $B_{1}=\left|\arctan M_{1}-\arctan M_{3}\right|<\delta, B_{2}$ $=\left|\arctan M_{2}-\arctan M_{3}\right| \leqslant \delta$ where $B_{1}$ and $B_{2}$ are the angles made by $L$ and $L_{1}$ and by $L$ and $L_{2}$ respectively. If $L_{1} \cap L_{2}=\left\{z^{*}\right\}$ is the apex of the triangle formed by $L_{1}, L_{2}$ and $L_{3}$ then the altitude to $L$ from $z^{*}$ has length $h$ where

$$
h \leqslant N / 2 \tan \delta .
$$

Note that in the degenerate case we can assume $h=0$. Hence, for all $z(s) \in \Gamma$ with $s_{1}<s<s_{2}$, we have $d(z(s), L) \leqslant h$.

For each $z(s)$ with $s>s^{\prime}$ we can find a chord to $\Gamma$ denoted $L$ of length $N$, such that the perpendicular bisector of $L$ passes through $z(s)$, with $z(s)$ between the endpoints of $L$. To complete the proof of Fact $\mathrm{A}$, we let $\delta$ be small enough so that $N / 2 \tan \delta<\varepsilon$ and choose $s^{\prime}$ as in (20). Clearly, similar arguments apply to $s<0$. So that repeating the above argument, mutatis mutandis, we find $s^{\prime \prime}<0$. Finally we conclude the proof of Fact A by letting $s_{0}=\operatorname{Max}\left(s^{\prime},\left|s^{\prime \prime}\right|\right)$.

We now claim the following is true:

FACT B. For all $r>0, \varepsilon>0$, there exists $s^{*}>0$ such that for all $s$ with $|s|>s^{*}$ there exists a circle of radius whose center depends on $z(s)$ and $\varepsilon$ such that (a) $d(z(s), C)<\varepsilon$, (b) $C$ is inside the convex domain bounded by $\Gamma$.

To prove Fact B, let $s_{0}$ be chosen as in Fact A where $N=2 r$. For each $s$, $|s|>s_{0}$ let the chord found in Fact A be denoted $L_{s}$. Define $C_{s}$ as the circle of radius $r$ which is tangent to $L_{s}$ at the midpoint of $L_{s}$ with the property that $C_{s}$ lies outside the compact region bounded by $L_{s}$ and a subarc of $\Gamma$. Since $\Gamma$ is not confined to any vertical strip, there exists $s^{*}>S_{0}$ such that $C_{s}$ is inside $\Gamma$ for $|s|>s^{*}$.

For $s$ fixed, let $z_{0}$ be the center of $C_{s}$ and consider the ring region $\Delta$ defined by: $r<\left|z-z_{0}\right|<R$ where

$$
\frac{1}{R(\log R / r)}=\lambda \text {. }
$$

Fact (iii) assures us that $\Delta \cap \mathscr{D} \subset \omega$. It is easy to show (22) is equivalent to

$$
R-r=R-R \cdot e^{-1 / \lambda R} \text {. }
$$

From this we see that

$$
\lim _{R \rightarrow \infty}(R-r)=1 / \lambda \text {. }
$$

Since by choosing $r$ sufficiently large we can be assured that $R$ and $\left|s^{*}\right|$ are as large as we please, the proof is now complete.

REMARK. The methods give no information about the free boundary when 
$\Gamma$ is confined to a vertical strip. For $\lambda>0$, we let $\omega_{\lambda}$ denote the constructed solution strip and let $\alpha_{\lambda}$ denote the free boundary of $\omega_{\lambda}$. We now discuss the properties of the constructed solution strip as $\lambda$ varies.

THEOREM 5. Let $\Gamma$ be an infinite convex fixed boundary with $\Gamma$ asymptotic to a pair of nonparallel straight lines. Then, (a) $\lambda_{1}>\lambda_{2}$ implies $\omega_{\lambda_{1}} \subset \omega_{\lambda_{2}}$, (b) $\cup_{\lambda>0} \alpha_{\lambda}=\mathscr{D}$.

ProOF. (a) is an immediate consequence of (iv) and (v). To show part (b) let $z_{1} \in \mathscr{D}$ and define

$$
A=A\left(z_{1}\right)=\left\{\lambda: z_{1} \in \omega_{\lambda}\right\}, \quad B=B\left(z_{1}\right)=\left\{\lambda: z_{1} \notin \omega_{\lambda}\right\} .
$$

We will show that neither $A$ nor $B$ is empty.

Given $z_{1} \in \mathscr{D}$ we show that for all $r>0$ there is a circle $C_{r}$ inside $\Gamma$ with center $z_{r}$ and radius $r$. Choose $\lambda$ so small that $1 /\left|z_{1}\right|\left[\log \left(\left|z_{1}\right| / r\right)\right]>\lambda$. By the minimality of $\omega_{\lambda}$ we see that $z_{1} \in \omega_{\lambda}$ and $A \neq \varnothing$.

To show $B$ is not empty, we recall that $\omega_{\lambda} \subset\{z \in \mathscr{D}: d(z, \Gamma)<1 / \lambda\}$. Hence if $z_{1} \in \mathscr{D}$ choose $\lambda$ such that $1 / \lambda<d\left(z_{1}, \Gamma\right)$ and $z_{1} \notin \omega_{\lambda}$.

Now let $\Omega_{1}=\bigcap_{\lambda \in A} \omega_{\lambda}, \Omega_{2}=\cup_{\lambda \in B} \omega_{\lambda}$. If $\Omega_{1}^{0}$ is the interior of $\Omega_{1}$ then both $\Omega_{1}^{0}$ and $\Omega_{2}$ are solutions for some $\lambda_{1}$ and $\lambda_{2}$ respectively. If $\lambda_{1} \neq \lambda_{2}$, then we apply a similar argument to that used in $[5$, p. 843] to obtain a contradiction. From this (b) follows.

3. Other regions. We say $\Gamma$ is an admissible curve if $\Gamma$ is described by $x+i f(x)$ where $f(x)$ is a continuous bounded function which has bounded Dini derivates at every point. In this section we give similar results regarding the free boundaries for admissible curves. We let $\mathscr{D}=\{z=x+i y: y>$ $f(x)$ \} and show that free boundaries do exist in $\mathscr{D}$ for admissible curves $\Gamma$.

THEOREM 6. Let $\Gamma$ be an admissible curve. If $Q \equiv \lambda$ a positive constant in (3), then there exists $\omega \in \mathcal{C}(\Gamma)$ with the properties that $V_{\omega}$ satisfies (1), (2) and (3). Furthermore, if $\alpha$ is the free boundary of $\omega$, then every vertical line intersects $\alpha$ at exactly one point.

Proof. Let $R_{n}$ be the closed strip $-n \leqslant x \leqslant+n$ and let $\Gamma_{n}=\Gamma \cap R_{n}$. Let

$$
\begin{aligned}
& \bar{m}_{n}^{*}=\sup \left\{\frac{f(s)-f(t)}{s-t}: s, t \in[-n, n]\right\}, \\
& \underline{m}_{n}^{*}=\inf \left\{\frac{f(s)-f(t)}{s-t}: s, t \in[-n, n]\right\},
\end{aligned}
$$

which are finite by hypothesis. Let

$$
\bar{m}_{n}=\max \left\{\bar{m}_{n}^{*}+1, n\right\}, \quad \underline{m}_{n}=\min \left\{\underline{m}_{n}^{*}+1, n\right\} .
$$

If $r_{n}$ is the ray with slope $\bar{m}_{n}$ originating at the point $n+i f(n)=A_{n}$, then $r_{n}$ does not intersect $\Gamma_{n}$ except at the point $A_{n}$. Similarly, if $r_{-n}$ is the ray 
originating at the point $-n+i f(-n)=B_{n}$ with slope $\underline{m}_{n}$, then $r_{-n}$ does not intersect $\Gamma_{n}$ except at the point $B_{n}$. If $P_{n}$ is the point of intersection of lines $r_{n}$ and $r_{-n}$, then $P_{n} \notin \mathscr{D} \cup \Gamma$. Let $Q_{-n}$ and $Q_{n}$ denote the points of intersection of the rays $r_{n}$ and $r_{-n}$ with the lines $x=-n$ and $x=+n$ respectively. Let $\Gamma_{n}^{*}$ denote the curve defined by $\Gamma_{n}$ together with the line segments $\left[A_{n}, Q_{n+1}\right],\left[Q_{-n}, Q_{n}\right]$ and $\left[Q_{-n}, B_{n}\right]$. If $P$ lies in the triangle determined by the three points $Q_{n}, Q_{-n}$ and $P_{n}$ and $Q$ lies on the curve $\Gamma_{n}^{*}$, then the segment $[P, Q]$ lies outside of $\mathscr{D}$.

In the set $\mathcal{C}\left(\Gamma_{n}^{*}\right)$ there is a unique solution denoted by $\omega_{\lambda}^{(n)}$ with free boundary $\boldsymbol{\alpha}_{\lambda}^{(n)}$.

Using ideas quite similar to those used in the proof of Theorem 1, we could show that

$$
\omega=\bigcup_{n}\left(\omega_{\lambda}^{(n)} \cap \Phi\right)
$$

is a solution. Furthermore, since the free boundary of $\omega$ is an analytic curve and all points inside the triangle determined by the points $Q_{n}, Q_{-n}$, and $P_{n}$ are star center points for the curves $\alpha_{\lambda}^{(n)}$ for each $n$, we see that every vertical line intersects the free boundary of $\omega$ in exactly one point.

If $\Gamma$ is admissible, the region $\omega \in \mathcal{C}(\Gamma)$ whose existence is shown in Theorem 6, will be called the constructed solution strip. The following theorem gives qualitative properties about the free boundary of $\omega$.

THEOREM 7. Let $\Gamma$ be admissible and let $\omega$ be the constructed solution strip in $\mathcal{C}(\Gamma)$. If $\Gamma$ is asymptotic to the line $y=0$, then, $\alpha$, the free boundary of $\omega$, is asymptotic to the line $y=1 / \lambda$.

Proof. Given $\varepsilon>0$ and $r>0$ there exists $M>0$ such that for each $z_{0}$ where $\left|\operatorname{Re} z_{0}\right|>M$ and $\operatorname{Im} z_{0}=-(r+\varepsilon)$, the circle

$$
C_{r}\left(z_{0}\right)=\left\{z:\left|z-z_{0}\right|=r\right\}
$$

lies below $\Gamma$. Hence for $z \in \alpha$ and $|\operatorname{Re} z|>M$, we have $\operatorname{Im} z>\rho$ where

$$
(\rho+\varepsilon+r) \exp \left[\frac{-1}{\lambda(\rho+\varepsilon+r)}\right]=r .
$$

It follows that given $\delta>0$ for $|\operatorname{Re} z|$ sufficiently large, $|\operatorname{Im} z|>1 / \lambda-\delta$.

Since $\Gamma$ is admissible we let $\Gamma=\{x+i y: y=f(x)\}$ and $|f(x)|<k$. Furthermore since $\Gamma$ is asymptotic to the $x$-axis given $\varepsilon>0$ there exists $R>0$ such that $|\operatorname{Im} f(z)|<\varepsilon$ for $|x|>R$. Let $\Gamma_{n}^{*}$ be the curve which bounds the region

$$
\Delta_{n}=[\{z:|\operatorname{Re} z|<r,|\operatorname{Im} z|<k\} \cup\{z:|\operatorname{Re} z|<\varepsilon\}] \cap\{z:|\operatorname{Re} z|<n\} .
$$

Let $\mathscr{D}_{n}$ denote the convex hull of $\Delta_{n}$ and let $k_{n}=\partial \mathscr{D}_{n}$. If $\omega_{n}$ is the unique solution in $\mathcal{C}\left(k_{n}\right)$, then a simple application of (iv) shows that if $z \in \alpha$ and 
$|\operatorname{Re} z|=n$, then

$$
|\operatorname{Im} z|<\varepsilon+\left[\frac{\sqrt{(n-r)^{2}+(k-\varepsilon)^{2}}}{(n-r)}\right] \frac{1}{\lambda} .
$$

Therefore given $\delta>0$ for $|\operatorname{Re} z|$ sufficiently large, $|\operatorname{Im} z|<\delta+1 / \lambda$.

We conclude with the remark that one can use any family which is elliptic in the sense of [4] to study unbounded free boundary problems provided there is some control on the free boundary as the fixed boundary becomes unbounded. An example would be the following theorem whose proof we omit.

THEOREM 8. Let $\Gamma$ be an infinite starlike fixed boundary such that (1) $\{x$ : $x=\operatorname{Re} z, z \in \Gamma\}$ is unbounded above and below, and (2) $\{y: y=\operatorname{Im} z$, $z \in \Gamma\}$ is bounded above and below. Then, there exists a region $\omega \in \mathcal{C}(\Gamma)$ such that $V_{\omega}$ satisfies (1), (2) and (3) where $Q \equiv \lambda$ a fixed positive constant. Furthermore, the free boundary of $\omega$ is starlike.

\section{REFERENCES}

1. A. Acker, Heat loss inequalities, SIAM J. Math. Anal. 8 (1977).

2. (1977), 230-234.

3. A. Beurling, An extension of the Riemann mapping theorem, Acta Math. 90 (1953), 117-130.

4. Free boundary problems for the Laplace equation, Institute for Advanced Study Seminar, Princeton, N. J., 1957, pp. 248-263.

5. D. E. Tepper, Free boundary problem, SIAM J. Math. Anal. 5 (1974), 841-846.

6. , On a free boundary problem, the starlike case, SIAM J. Math. Anal. 6 (1975), $503-505$.

Department of Mathematics, Clark University, Worcester, Massachusetts 01610

Current address (D. E. Tepper): Department of Mathematics, Baruch College (CUNY), New York, New York 10010

Current address (Gerald Wildenberg): Department of Mathematics, University of Hartford, West Hartford, Connecticut 06117 